\title{
Selection of Surgical Candidates
}

Last Updated: July 13, 2021

\section{Introduction}

Medical management with anti-epileptic drugs (AEDs) is the first-line intervention for patients with epilepsy. However, $30-40 \%$ of patients have seizures that cannot be adequately controlled by AEDs. ${ }^{1}$ Drug-resistant epilepsy (DRE) is diagnosed when seizures persist after two or more appropriate AED trials. ${ }^{2}$ DRE is associated with impaired quality of life as well as higher rates of depression, anxiety, suicidality, and long-term mortality. ${ }^{3,4}$ As a group, patients with DRE account for $~ 80 \%$ of all epilepsy-related costs in the US. ${ }^{5,6}$

Surgical resection of the epileptogenic zone is the most effective treatment option for controlling seizures and improving life quality in patients with DRE. ${ }^{7}$ Several randomized controlled trials (RCTs) and metaanalyses demonstrate that epilepsy surgery offers the best chance of seizure control when $\geq 2$ AED trials have failed. ${ }^{8-11}$ Although postsurgical seizure freedom rates vary with many patient factors, approximately 40$80 \%$ of patients with DRE who undergo epilepsy surgery enjoy sustained seizure relief compared to $<10 \%$ of those receiving continued AED therapy. ${ }^{12-14}$

Prior to epilepsy surgery, patients undergo a comprehensive assessment to determine their surgical candidacy and to plan the intervention. This socalled "presurgical evaluation" comprises a series of clinical and neurodiagnostic studies aimed at localizing the seizure foci as well as eloquent brain regions. An accurate and comprehensive presurgical evaluation maximizes the likelihood of seizure freedom and mitigates the risks associated with brain surgery. The purpose of this chapter is to highlight 
the main steps and modalities that constitute the presurgical evaluation for focal epilepsy surgery.

\section{Identification of Surgical Candidates}

\section{Surgical Indications}

The first step of the presurgical evaluation is to identify patients who should be referred for surgical assessment. With the exception of surgical referral for evaluation of treatment-resistant, disabling complex partial seizures, ${ }^{15}$ there are no well-established indications for epilepsy surgery evaluation.

In general, patients with DRE suffering from intractable, focal seizures should be referred for surgical evaluation. ${ }^{16}$ While this indication is broad, evidence suggests that only a fraction of potential epilepsy surgery candidates receive a formal surgical consultation. ${ }^{17,18}$ Considering the risks that accumulate with chronic seizures and AED therapy, many have advocated for lower referral thresholds in an effort to increase utilization and avail more patients to the potential benefits of epilepsy surgery. ${ }^{19}$

According to epileptologists Philippe Ryvlin and Sylvain Rheims, ${ }^{20}$ patients should be considered for epilepsy surgery when the following 3 conditions are met:

1. "The patient (or his or her parents for young children and patients with intellectual impairment) needs to understand the objective of the presurgical evaluation and to agree on the possibility of a surgical treatment."

2. "The patient should suffer from disabling seizures despite appropriate medical therapy."

Most patients referred for surgical evaluation qualify for the diagnosis of DRE. In 2010, the International League Against Epilepsy (ILAE) released a consensus statement defining DRE as the "...failure to achieve sustained seizure freedom with adequate trials of at least 
two appropriately chosen and used AED regimens (whether administered as monotherapies or in combination)."

The possibility of AED pseudo-resistance must be excluded before surgery is considered. Situations mimicking AED resistance include: misdiagnosis of epilepsy, inappropriate AED selection, inadequate dosage, drug interactions, and limited adherence to the medication regimen. ${ }^{21}$

While lack of adherence to AED therapy (e.g. due to side effects) does not contribute to the diagnosis of DRE, poor medication tolerance could justify the decision to pursue epilepsy surgery in the absence of formally demonstrated drug resistance.

3. "Available imaging and electro-clinical data should be consistent with the possibility of a surgically remediable epileptic syndrome."

The notion of a surgically-remediable epilepsy syndrome refers to a pattern of epilepsy that is clinically stereotyped, frequently resistant to AEDs, and responsive to surgery. ${ }^{22}$ The prototypical example of a surgically-remediable epilepsy syndrome is temporal lobe epilepsy associated with mesial temporal sclerosis (MTS). MTS is the most common cause of focal epilepsy in adults and is often refractory to medications. ${ }^{23}$ The histopathologic substrate of MTS is progressive gliosis and neuronal loss within the hippocampal subfields. ${ }^{24}$ Patients often present with intractable complex partial seizures associated with visual, olfactory, or gustatory auras. Over time, kindling of the contralateral temporal lobe through repeated propagation can produce partially-independent, bilateral seizure foci.

Surgical management of MTS typically involves either standardized or tailored anterior temporal lobe resection or laser ablation. In 2001, a ground-breaking prospective RCT by Wiebe and colleagues 8 demonstrated that $\sim 60 \%$ of patients with MTS were seizure-free 
one year after mesial temporal lobe surgery compared with only $8 \%$ of patients receiving continued pharmacotherapy. Surgery was also associated with superior quality of life. Subsequent studies have documented seizure-freedom rates after anterior temporal lobe resection as high as $70-85 \%$ in patients with MTS. ${ }^{13,25}$

In addition to MTS, other surgically-remediable epilepsy syndromes include various hemispheric disorders (e.g., Rasmussen's encephalitis, hemimegencephaly, Sturge-Weber syndrome, hemispheric encephalomalacia) and forms of lesional epilepsy (e.g., tumor-related epilepsy, vascular aberrations, malformations of cortical development, temporal encephalopathies, tuberous sclerosis). ${ }^{22,26}$ Detailed discussion of these entities, as well as the surgical options for patients with non-focal epilepsy syndromes (e.g., corpus callosotomy, vagus nerve stimulation, and deep brain stimulation) is beyond the scope of this chapter. The remainder of the text focuses on the evaluation for focal epilepsy surgery.

\section{Referral to an Epilepsy Center}

Epilepsy surgery candidates should be referred to a specialized Epilepsy Center. The National Association of Epilepsy Centers (NAEC) recognizes four accreditation levels of epilepsy care based on the range of offered services and the quality of the facilities and personnel. Level 4 Epilepsy Centers offer the full range of complex surgical procedures and intracranial monitoring techniques, making them best equipped for the evaluation of surgical candidates. A directory of Level 4 Epilepsy Centers is available online.

\section{Presurgical Evaluation: Concepts and Implementation}

\section{Overview}

Before a patient can be offered epilepsy surgery, two questions must be answered: (1) Can the seizure foci be confidently localized? (2) Do the anticipated benefits of surgery outweigh the risks? In principle, these 
questions hinge on the accurate delineation of the epileptogenic zone, or the "minimum amount of cortex that must be resected (inactivated or completely disconnected) to produce seizure freedom," 27 as well as eloquent brain regions that must be spared to ensure an acceptable functional outcome.

During the presurgical evaluation, various clinical and neuro-diagnostic tools can be leveraged to tailor a surgical plan that maximally targets the epileptogenic zone while minimizing damage to key functional circuits. All tests, from the history to advanced imaging, essentially assess one or both of the following concepts:

1. Where is the epileptogenic zone?

2. What deficits may be incurred by resection or ablation of this zone?

\section{Essential Non-Invasive Modalities}

Upon referral, all patients undergo a non-invasive investigation consisting of a clinical assessment and various neuro-diagnostic studies. Detailed review of epilepsy history, seizure semiology/symptomatology, and neurological examination provides initial insights into the underlying etiology and seizure circuitry. Non-invasive tests are used to further refine the diagnostic assessment by revealing the distribution of structural and electrographic abnormalities.

Essential components of the non-invasive evaluation include:

- Interictal scalp recordings: Scalp electrodes placed in the standard 10-10 or 10-20 configuration provide a record of neural activity with excellent temporal resolution. Patients typically begin with a short ( 30-60 minute) recording session to detect interictal (i.e., between-seizure) signal abnormalities.

Hemispheric asymmetries in the background oscillatory characteristics of the EEG (e.g., hemispheric slowing or disorganization/loss of the posterior-anterior spectral gradient) can 
provide useful lateralizing information, whereas focal abnormalities (e.g., interictal spikes, spike/slow wave complexes, focal slowing, signal attenuation) are suggestive of underlying pathology within the recording field of the electrode. The distribution of interictal abnormalities could also raise concern for a multifocal or generalized epilepsy syndrome, which has critical implications for surgical candidacy.

Limitations of interictal scalp recordings relate to the distortion of the signal as it travels from the brain to the surface electrode. Among many sources of impedance, the skull profoundly distorts the signal and can lead to localization errors. Muscle artifact from temporalis and facial contraction adds noise to the recording, especially during motor seizures. Additionally, certain epileptiform sources are notoriously difficult to localize using scalp electrodes, including those within the insula, midline sagittal, and inferior cortical regions. ${ }^{28}$

- Long-term video-EEG monitoring: The goal of prolonged scalp EEG monitoring (e.g., >24 hours) with synchronized video is to establish a correlation between the patient's ictal EEG activity and any clinical seizure manifestations. Provocative maneuvers such as AED withdrawal, sleep deprivation, exercise, hyperventilation, and photic stimulation can be employed to increase the chances of capturing multiple examples of the patient's debilitating seizure types. ${ }^{28}$

Interpretation of ictal EEG activity must be performed in the context of time-locked signs and symptoms. For instance, significant delay between the earliest clinical manifestations and the electrographic onset suggests that the seizure has propagated from an unmeasured source. Other limitations of ictal EEG analysis include the inaccessibility of certain seizure foci to scalp electrodes (e.g., mesial frontal, orbito-frontal, and occipital foci), the tendency for ictal activity to rapidly propagate to inter-dependent brain regions (which may lead to false-positive localization), and signal obscuration from muscle and movement artifact. 
The ideal surgical candidate exhibits a consistent and stereotyped electrographic ictal onset pattern that is concordant with the semiology, symptomatology, interictal abnormalities, and if present, the suspicious anatomic lesion. When these data are discordant or otherwise inconclusive, invasive exploration with intracranial electrodes may be warranted (see Chapter on Intracranial Monitoring). In some cases, the ictal onset as measured by scalp electrodes appears diffuse whereas subsequent invasive monitoring identifies a focal seizure origin. Thus, generalized ictal onset on scalp EEG should not automatically disqualify patients from surgical consideration, especially when other data suggest a focal epileptogenic process.

- High-resolution MRI: Magnetic resonance (MR) imaging is used to anatomically localize potential epileptogenic lesions. Most epilepsy centers have dedicated temporal and extra-temporal epilepsy protocols featuring 3 Tesla magnets, high soft tissue contrast, and thin slice thickness. ${ }^{29}$ In general, epilepsy protocols incorporate volumetric T1-weighted images, axial and sagittal T2-weighted images, axial diffusion weighted images (DWI), coronal and threedimensional fluid-attenuated inversion recovery (FLAIR) sequences, and coronal T2-weighted gradient echo sequence (GRE). ${ }^{30}$ These sequences improve the opportunity to detect subtle malformations of cortical development.

Well-equipped centers may incorporate other three-dimensional volumetric sequences with improved signal-to-noise ratio, such as magnetization-prepared rapid acquisition gradient echo (MP-RAGE) and fast spoiled gradient-recalled echo (FSPGR). Even with sensitive protocols, certain lesional pathologies can be difficult or impossible to detect with MRI (e.g., small areas of focal cortical dysplasia). The absence of a potentially epileptogenic lesion on MRI reduces the likelihood of a seizure-free outcome after epilepsy surgery, and this has an influence on surgical candidacy. ${ }^{31-33}$ 


\section{Ancillary Non-Invasive Modalities}

Ancillary modalities can be used to obtain complementary structural, functional, electrographic, and metabolic data on a case-by-case basis. Like the history, physical exam, EEG, and MRI, these can be grouped by whether they attempt seizure localization, functional localization, or both.

\section{Seizure Localization}

- SPECT: Seizure focus localization with ictal single photon emission computed tomography (SPECT) rests on the premise that the seizure focus receives increased blood flow during seizures relative to uninvolved brain regions.

- PET: Interictal regional hypo-metabolism revealed by positron emission tomography with fluorodeoxyglucose ( $\left.{ }^{18} \mathrm{FDG}-\mathrm{PET}\right)$ can be used to assess epileptogenic zones. PET has been shown to lateralize the epileptogenic temporal lobe in a majority of patients with TLE. 34,35

\section{Functional Localization}

- Functional MRI (fMRI): Functional mapping with $\mathrm{fMRI}$ is achieved by measuring the blood oxygen-level dependent (BOLD) signal while patients engage in functional tasks. The hypothesized location of the epileptogenic zone and the proposed resection margins dictate which functional domains are tested. For example, when the resection plan encroaches on the peri-rolandic cortex, motor and somatosensory mapping can be performed to define safe resection limits, especially in the setting of atypical gyral anatomy. Language tasks can be performed to lateralize language function and to demonstrate the spatial relationship of key language centers in relation to lesions or other potential epileptogenic zones.

A key limitation of $\mathrm{fMRI}$ is the difficulty of distinguishing between "essential" and "non-essential" patterns of taskdependent activation. ${ }^{36}$ For example, language tasks activate not only the critical language hubs, but also non-specific 
circuits involved in attention and general cognitive processes. This can lead to uncertainty when determining which regions of the functional circuit can or cannot be excised.

- Wada test: Before the popularization of fMRI, the Wada test (i.e., intracarotid sodium amobarbital procedure) was routinely used to lateralize domains of language and memory. ${ }^{36}$ In patients with left TLE, language lateralization with $\mathrm{fMRI}$ and Wada can produce discrepant results, with fMRI typically measuring higher degrees of right-sided language representation than observed on Wada testing.37Interestingly, studies involving patients with left TLE have shown that the degree of right-sided language representation measured by $\mathrm{fMRI}$ predicts the extent of language recovery after left ATL, a relationship that was less apparent when measuring language dominance with the Wada test. $^{38,39}$

Considering the widespread availability and favorable risk profile of $\mathrm{fMRI}$, many centers have moved away from the Wada test for routine cases of medial TLE. This practice is supported by a 2017 guideline released by the American Academy of Neurology (AAN), which states that fMRI may be used in place of the Wada test for lateralization of language and memory functions in patients with TLE. 40

3. Seizure and Functional Localization

- Magnetoencephalography (MEG): MEG is a non-invasive technique used to measure small magnetic fields generated by synchronized electrical currents in the brain. ${ }^{41}$ Compared to EEG, MEG offers superior spatial resolution due to the large number of recording sensors and the relative absence of signal distortion by intervening tissues. The interpretation of interictal MEG waveforms is similar to EEG, as the two technologies measure the same electrical phenomena. ${ }^{41}$ However, MEG offers the advantage of three-dimensional 
dipole modeling that is not compromised by the distorting effects of the scalp, meninges, and skull. For this reason, MEG has the potential to uncover a well-localized dipole cluster as the source of interictal spike activity that may be diffusely present in scalp EEG recordings. This can be helpful in planning the placement of intracranial electrodes or to confirm the epileptogenic potential of the lesions found on MRI. MEG can also be used to in the functional domain to localize motor, sensory, and language circuits.

- Transcranial magnetic stimulation (TMS): TMS is a noninvasive technology used to perform functional mapping. During the TMS procedure, a rapidly-changing magnetic field is produced by a coil placed on the scalp, causing temporary inactivation of the target region. Unlike fMRI, TMS can discern between "essential" and "non-essential" hubs based on the presence or absence of functional arrest in response to stimulation, respectively. Further, when thresholds for motor evoked potentials are higher on one side than the other, this can support ipsilateral seizure onset.

\section{Multi-disciplinary case conference}

After completion of the non-invasive evaluation, patients are discussed at a multi-disciplinary case conference involving experts from neurology, neurosurgery, radiology, neuropsychology, nursing, and social work. ${ }^{42}$ The goal of the conference is to distinguish between the following treatment paths on the basis of non-invasive findings:

1. The patient is not a candidate for epilepsy surgery due to the presence of multi-focal seizures or generalized epilepsy. For such patients, palliative therapies such as vagus nerve stimulation (VNS) or ketogenic diet can be considered, in addition to further medical management.

2. The patient is a surgical candidate and can proceed directly to surgery. This recommendation is made when concordant non- 
invasive findings point to a well-circumscribed epileptogenic focus that can be safely excised without compromising key functional circuits.

3. The patient is a surgical candidate but requires further evaluation. These patients are recommended for invasive evaluation due to some degree of uncertainty or discordance in the non-invasive work-up, or in the setting of concordant data with a non-lesional MRI, to define the limits of a resection. Approximately $30-40 \%$ of patients must undergo invasive monitoring with intracranial electrodes before resective surgery can be performed. ${ }^{43}$ The major approaches for long-term invasive EEG monitoring are stereoelectroencephalograpy (SEEG) and subdural grid/strip electrodes. While a patient is implanted, functional and epileptogenic mapping can also be performed by delivering current through invasive electrodes. For a detailed discussion of the indications, techniques, safety, tolerability, and outcomes of invasive EEG, please refer to the dedicated Intracranial Monitoring chapter.

\section{Conclusions}

- Most patients with debilitating, focal-onset seizures should be referred for surgical evaluation.

- All surgical candidates should undergo a non-invasive evaluation consisting of clinical assessment, scalp EEG, structural MRI, and various ancillary tests that can be incorporated on a case-by-case basis.

- Approximately $30-40 \%$ of patients will subsequently undergo an intracranial study to determine the intervention strategy.

Contributors: Samuel B. Tomlinson BA, Vivek P. Buch MD, Benjamin C. Kennedy MD

DOI: https://doi.org/10.18791/nsatlas.v7.ch01 
1. Kwan P, Brodie MJ. Early identification of refractory epilepsy. N Engl J Med 2000; 342: 314-319.

2. Kwan P, Arzimanoglou A, Berg AT, et al. Definition of drug resistant epilepsy: consensus proposal by the ad hoc Task Force of the ILAE Commission on Therapeutic Strategies. Epilepsia 2010; 51: 1069-1077.

3. Kwon O-Y, Park S-P. Depression and anxiety in people with epilepsy. Journal of clinical neurology (Seoul, Korea) 2014; 10: 175-188.

4. Sillanpää M, Shinnar S. Long-Term Mortality in Childhood-Onset Epilepsy. New England Journal of Medicine 2010; 363: 2522-2529.

5. Begley CE, Famulari M, Annegers JF, et al. The cost of epilepsy in the United States: an estimate from population-based clinical and survey data. Epilepsia 2000; 41: 342-351.

6. Cramer JA, Wang ZJ, Chang E, et al. Healthcare utilization and costs in adults with stable and uncontrolled epilepsy. Epilepsy Behav 2014; 31: 356-362.

7. Ryvlin $\mathrm{P}$, Cross $\mathrm{JH}$, Rheims S. Epilepsy surgery in children and adults. Lancet Neurol 2014; 13: 1114-1126.

8. Wiebe S, Blume WT, Girvin JP, et al. A randomized, controlled trial of surgery for temporal-lobe epilepsy. N Engl J Med 2001; 345: 311-318.

9. Engel J, McDermott MP, Wiebe S, et al. Early Surgical Therapy for Drug-Resistant Temporal Lobe Epilepsy: A Randomized Trial. JAMA 2012; 307: 922-930.

10. Dwivedi R, Ramanujam B, Chandra PS, et al. Surgery for DrugResistant Epilepsy in Children. N Engl J Med 2017; 377: 1639-1647.

11. Liu JT, Liu B, Zhang H. Surgical versus medical treatment of drugresistant epilepsy: A systematic review and meta-analysis. Epilepsy Behav 2018; 82: 179-188.

12. Chen Z, Brodie MJ, Liew D, et al. Treatment Outcomes in Patients 
With Newly Diagnosed Epilepsy Treated With Established and New Antiepileptic Drugs: A 30-Year Longitudinal Cohort Study. JAMA Neurol 2018; 75: 279-286.

13. Spencer S, Huh L. Outcomes of epilepsy surgery in adults and children. Lancet Neurol 2008; 7: 525-537.

14. Kwan P, Brodie MJ. Drug treatment of epilepsy: when does it fail and how to optimize its use? CNS Spectr 2004; 9: 110-119.

15. Engel J, Wiebe S, French J, et al. Practice parameter: Temporal lobe and localized neocortical resections for epilepsy. Report of the Quality Standards Subcommittee of the American Academy of Neurology, in Association with the American Epilepsy Society and the American Association of Neurological Surgeons 2003; 60: 538-547.

16. Miller JW, Hakimian S. Surgical treatment of epilepsy. Continuum (Minneapolis, Minn.) 2013; 19: 730-742.

17. Engel J, Jr. A Greater Role for Surgical Treatment of Epilepsy: Why and When? Epilepsy currents 2003; 3: 37-40.

18. Steinbrenner M, Kowski AB, Holtkamp M. Referral to evaluation for epilepsy surgery: Reluctance by epileptologists and patients. Epilepsia 2019; 60: 211-219.

19. Engel J, Jr., Wiebe S, Radhakrishnan K, et al. Surgical treatment for epilepsy. Neurologisch : fachmagazin fur neurologie 2013; 2013: 12-14.

20. Ryvlin P, Rheims S. Epilepsy surgery: eligibility criteria and presurgical evaluation. Dialogues Clin Neurosci 2008; 10: 91-103.

21. Wiebe $S$, Jetté N. Issues of Medical Intractability for Surgical Candidacy. In Wyllie E, Gidal BE, Goodkin HP, et al. (Eds) Wyllie's Treatment of Epilepsy: Principles and Practice, Wolters Kluwer: Philadelphia, PA; 2015:789-793.

22. Rathore C, Radhakrishnan K. Concept of epilepsy surgery and 
presurgical evaluation. Epileptic Disord 2015; 17: 19-31; quiz 31.

23. Englot DJ, Chang EF. Rates and predictors of seizure freedom in resective epilepsy surgery: an update. Neurosurg Rev 2014; 37: 389-404; discussion 404-385.

24. Thom M. Review: Hippocampal sclerosis in epilepsy: a neuropathology review. Neuropathology and applied neurobiology 2014; 40: 520-543.

25. Englot DJ, Lee AT, Tsai C, et al. Seizure Types and Frequency in Patients Who "Fail" Temporal Lobectomy for Intractable Epilepsy. Neurosurgery 2013; 73: 838-844.

26. Engel J. Overview of Surgical Treatment for Epilepsy. In Shorvon S, Perucca E, Engel J (Eds) The Treatment of Epilepsy, Wiley-Blackwell: Hoboken, New Jersey; 2015:709-722.

27. Luders HO, Najm I, Nair D, et al. The epileptogenic zone: general principles. Epileptic Disord 2006; 8 Suppl 2: S1-9.

28. Britton JM. Video-EEG Monitoring in the Presurgical Evaluation. In Wyllie E, Gidal BE, Goodkin HP, et al. (Eds) Wyllie's Treatment of Epilepsy: Principles and Practice, Wolters Kluwer: Philadelphia, PA; 2015:810-823.

29. Moosa ANV, Ruggieri PM. Magnetic Resonance Imaging in Evaluation for Epilepsy Surgery. In Wyllie E, Gidal BE, Goodkin HP, et al. (Eds) Wyllie's Treatment of Epilepsy: Principles and Practice, Wolters Kluwer: Philadelphia, PA; 2015:794-809.

30. Miserocchi A, Nowell M, McEvoy AW. Neuroradiologic Evaluation for Epilepsy Surgery: Magnetic Resonance Imaging. In Winn HR (Ed) Youmans and Winn Neurological Surgery, Elsevier, Inc.: Philadelphia, PA; 2017:420432.

31. Tonini C, Beghi E, Berg AT, et al. Predictors of epilepsy surgery outcome: a meta-analysis. Epilepsy Res 2004; 62: 75-87.

32. Kuzniecky R, Burgard S, Faught E, et al. Predictive value of magnetic 
resonance imaging in temporal lobe epilepsy surgery. Arch Neurol 1993; 50: 65-69.

33. Chapman K, Wyllie E, Najm I, et al. Seizure outcome after epilepsy surgery in patients with normal preoperative MRI. J Neurol Neurosurg Psychiatry 2005; 76: 710-713.

34. Gaillard WD. Nuclear Imaging (PET, SPECT). In Wyllie E, Gidal BE, Goodkin HP, et al. (Eds) Wyllie's Treatment of Epilepsy: Principles and Practice, Wolters Kluwer: Philadelphia, PA; 2015:824-832.

35. Ho SS, Berkovic SF, Berlangieri SU, et al. Comparison of ictal SPECT and interictal PET in the presurgical evaluation of temporal lobe epilepsy. Ann Neurol 1995; 37: 738-745.

36. Binder JR, Carlson C. Language and Memory Mapping. In Wyllie E, Gidal BE, Goodkin HP, et al. (Eds) Wyllie's Treatment of Epilepsy: Principles and Practice, Wolters Kluwer: Philadelphia, PA; 2015:865-874.

37. Janecek JK, Swanson SJ, Sabsevitz DS, et al. Language lateralization by fMRI and Wada testing in 229 patients with epilepsy: rates and predictors of discordance. Epilepsia 2013; 54: 314-322.

38. Janecek JK, Swanson SJ, Sabsevitz DS, et al. Naming outcome prediction in patients with discordant Wada and fMRI language lateralization. Epilepsy Behav 2013; 27: 399-403.

39. Sabsevitz DS, Swanson SJ, Hammeke TA, et al. Use of preoperative functional neuroimaging to predict language deficits from epilepsy surgery. Neurology 2003; 60: 1788-1792.

40. Szaflarski JP, Gloss D, Binder JR, et al. Practice guideline summary: Use of $\mathrm{fMRI}$ in the presurgical evaluation of patients with epilepsy: Report of the Guideline Development, Dissemination, and Implementation Subcommittee of the American Academy of Neurology. Neurology 2017; 88: 395-402.

41. Burgess RC, Mosher JC. Magnetoencephalography. In Wyllie E, Gidal 
BE, Goodkin HP, et al. (Eds) Wyllie's Treatment of Epilepsy: Principles and Practice, Wolters Kluwer: Philadelphia, PA; 2015:833-847.

42. Feldman L, Kotagal P. Patient management conference: Correlating pre-surgical data. Seizure 2019.

43. Kovac S, Vakharia VN, Scott C, et al. Invasive epilepsy surgery evaluation. Seizure 2017; 44: 125-136. 